\title{
L'ingénieur de l'an 2000 - La formation continuée et l'hydraulique
}

\author{
N. Dehousse \\ Professeur à l'Université de Liège
}

\section{La formation continuée en général. Tentative de quantification de son importance}

\section{A. Capital immmatériel (tentative de quantification)}

1. Les bases de fonctionnement d'une entreprise sont triples : son capital financier, son capital matériel et son capital immatériel.

Notre propos ne concerne que ce dernier capital, les deux premiers étant aisément quantifiables et bien connus.

Le capital immatériel dont dispose une firme, comprend en gros:

a. son savoir faire,

b. les dépenses consenties pour le vendre (Marketing),

c. les dépenses consenties pour l'entretenir et le développer $(R$ et $D)$.

Les dépenses $b$ peuvent être chiffrées.

Cette opération est beaucoup moins évidente en ce qui concerne le savoir faire ou le capital intellectuel lequel est porté par les acteurs principaux de la firme.

Par acteurs principaux, nous entendons toutes les personnes qui sont chargées de responsabilités majeures parmi lesquelles figurent évidemment, aux premières loges, les ingénieurs.

Le propos qui suit vise à estimer, à la manière d'un économiste ou d'un actuaire, la valeur d'un de ces acteursingénieurs.

2. Le fondement du raisonnement repose sur l'estimation de la qualité des connaissances d'un ingénieur.

Ces connaissances sont de deux natures: les connaissances fondamentales et celles qui sont spécifiques à un secteur d'activité.

Parmi les connaissances fondamentales nous avons évidemment: la mathématique, la physique, la chimie, l'électricité, la mécanique, la gestion, les langues, ...

Le bagage spécifique est propre aux secteurs des mines, de la métallurgie, de la construction, ... de l'électronique, ...

Une estimation grossière basée sur les programmes universitaires conduit à $\pm 70 \%$ pour les premières et $30 \%$ pour les secondes. Le clivage ne correspond pas aux études de candidature ingénieur vis-à-vis de celles des études techniques.

Nombre de connaissances de base pour l'activité de l'ingénieur sont évidemment enseignées dans les trois années de spécialisation (en Belgique).
Ces connaissances fondamentales et spécifiques vont connaître le processus d'obsolescence: processus moins marqué pour les connaissances fondamentales que pour les connaissances spécifiques. Pour ces dernières, le taux de vieillissement et de remplacement sera d'autant plus accentué qu'il s'agit de domaines porteurs de nouveauté.

Les figures 1 et 2 schématisent des évolutions moyennes supputées d'après les hypothèses suivantes: l'ingénieur débute sa carrière à 25 ans et connaît une période d'activité de 40 ans; deux domaines spécifiques sont retenus, ceux représentatifs des activités industrielles traditionnelles (mines, métallurgie, construction, mécanique, ...) et ceux représentatifs des activités industrielles récentes (électronique, informatique, ....).

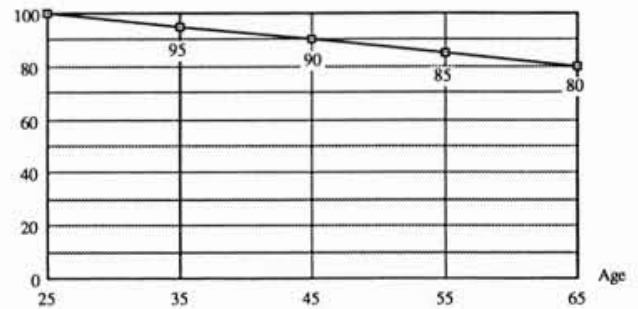

1. Diagramme $B$

Obsolescence naturelle des connaissances fondamentales pendant la durée de vie de l'ingénieur.

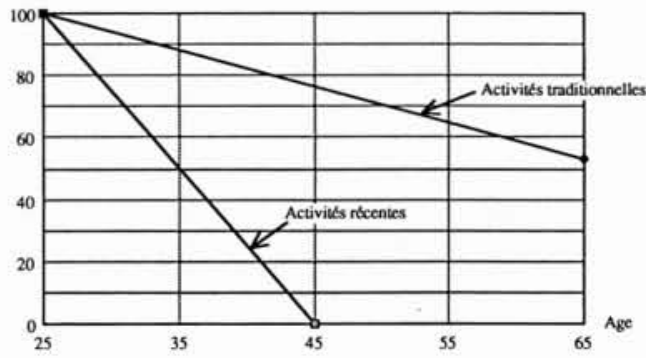

2. Diagramme $S$.

Obsolescence naturelle des connaissances spécifiques pendant la durée de vie de l'ingénieur. 
On comprendra d'emblée le caractère subjectif et discutable de ces diagrammes qui ne se justifient que par un désir d'obtention d'ordres de grandeur.

La figure 3 représente l'évolution du bagage total sur la base $0,7 \times B+0,3 \times S$.

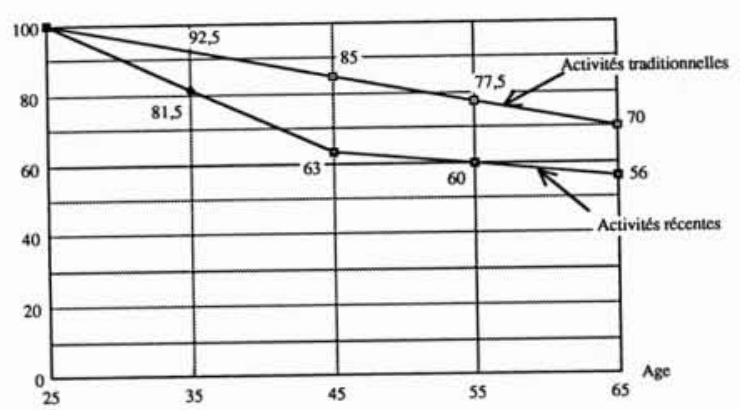

3. Diagramme $B+S$.

Obsolescence naturelle des connaissances globales pendant la durée de vie de l'ingénieur.

3. Indépendamment de l'obsolescence naturelle des connaissances qui dépend de l'évolution générale de la science et de la technique, l'acquis intellectuel connaît une déperdition normale inévitable due à l'absence de pratique (mémoire), au développemetn d'autres centres d'intérêt : familiaux, sociaux, culturels, sportifs, ... La figure 4 traduit ce phénomène.

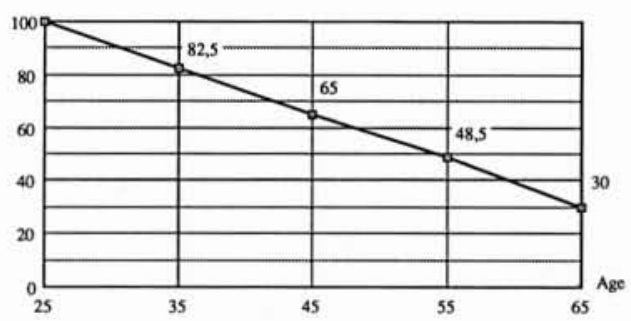

4. Diagramme $D_{l}$.

Déperdition normale des connaissances pendant la durée de vie de l'ingénieur.

Nous sommes ici dans le subjectif le plus total. Le choix fait est le reflet de relations personnelles.

4. Par contre, en fonction de l'âge croît l'expérience professionnelle en technique, organisation, information, langues, commerce, ....

Le diagramme évolutif suggéré est celui de la figure 5 .

Il est vraisemblable que, compte tenu des changements de postes au cours de la carrière ce diagramme devrait plutôt se présenter de manière discontinue $\left(E^{\prime}\right)$.

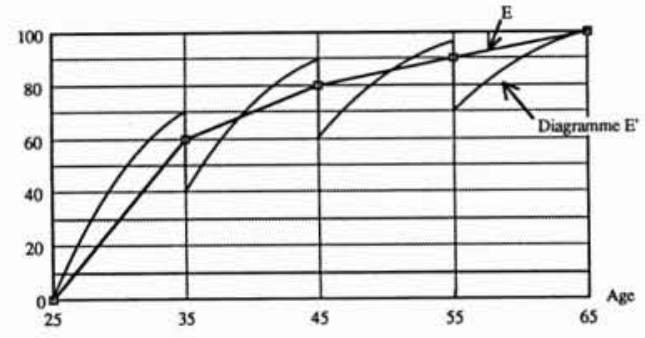

5. Diagramme E.

Evolution de l'expérience professionnelle.

Le diagramme $E$ peut être compris comme un lissage de ces discontinuités.

Nous conserverons le diagramme $E$ dans un souci de simplification.

Enfin le diagramme $E$ exige lui-même une correction de déperdition $D_{2}$ pour causes physiologiques (sénescence).

Ce dernier diagramme est extrait de données actuariales/ (Sadi Kirchen : Le prix d'un homme - Trends - mars 1989).

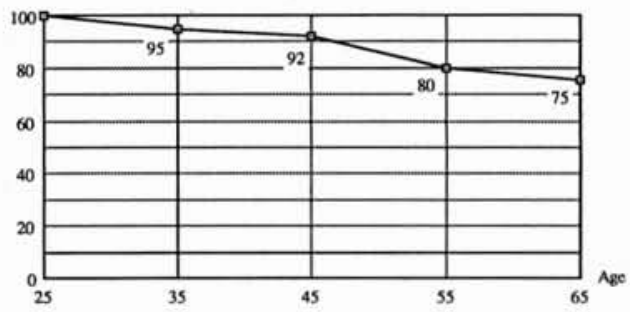

6. Diagramme $\mathrm{D}_{2}$.

Déperdition pour causes physiologiques.

5. Pour la quantification globale de la personnalité, estimation doit être faite de la part des connaissances et de celle de l'expérience professionnelle. La proportion 0,6 ; 0,4 a été admise.

$\mathrm{Si}$ bien que la valeur intellectuelle globale relative résultera de l'opération :

$$
\left[(B+S) \times D_{1} \times 0,6+E \times D_{2} \times 0,4\right] \frac{1}{0,6}
$$

Le résultat est fourni par les figures 7 et 8 pour les techniques traditionnelles et les techniques récentes et ce dans l'hypothèse où aucune formation complémentaire ou continuée n'a été acquise.

Si l'on admet l'évolution suivante des traitements en fonction de l'âge (coût à l'entreprise) :

- à 25 ans $1,3 \mathrm{M}$

- à 35 ans $3,0 \mathrm{M}$

- à 45 ans $3,5 \mathrm{M}$

- à 55 ans $4,0 \mathrm{M}$

- à 65 ans $4,5 \mathrm{M}$ 
(valeurs en francs courants, ...), on peut quantifier le capital intellectuel que représentent les cadres ingénieurs en multipliant les courbes $G_{1}$ et $G_{2}$ par les valeurs ci-avant.

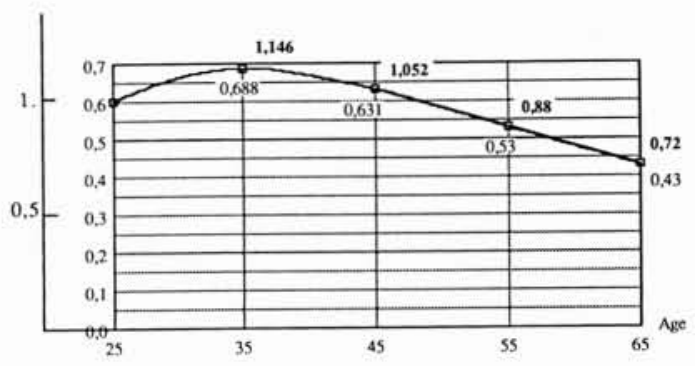

7. Diagramme $G_{f}$.

Estimation de la valeur intellectuelle relative (sans recyclage). Secteurs traditionnels.

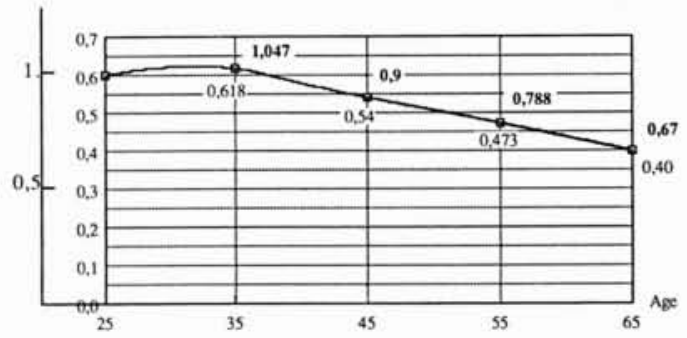

8. Diagramme $G_{2}$.

Estimation de la valeur intellectuelle relative (sans recyclage). Secteurs récents.

On obtient les réulstats suivants en dehors de tout recyclage :

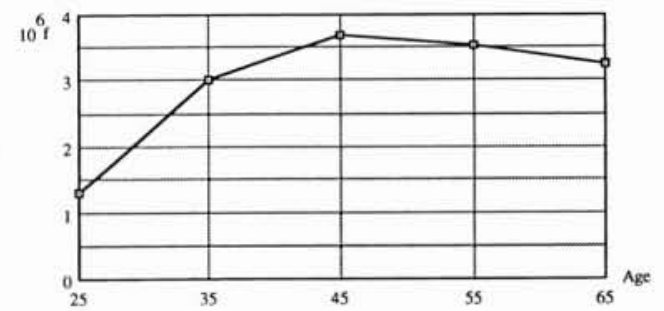

9. Estimation de la valeur intellectuelle (sans recyclage). Secteurs traditionnels.

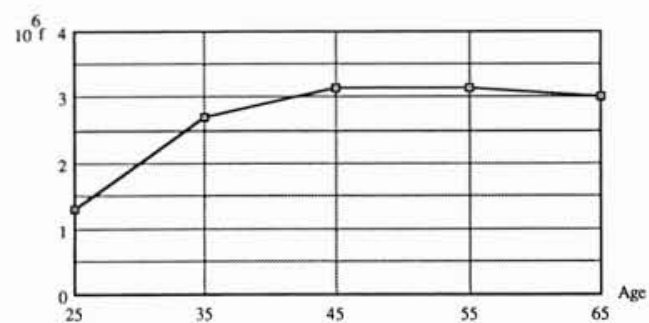

10. Estimation de la valeur intellectuelle (sans recyclage). Secteurs récents.
A ces valeurs, il y aurait lieu d'ajouter \pm 5 millions de coût de formation universitaire prise en charge par la société au sens large du terme.

L'importance de ces valeurs souligne toute la qualité du capital intellectuel d'une firme, le "drame " que représentent les départs en cours de carrière, les préretraites, les licenciements, ...

Elle explique aussi, qu'après un conflit une firme puisse repartir sans capital financier et sans capital matériel.

Une dernière conclusion s'impose, c'est que cette importance conduit à consentir des efforts pour entretenir et développer le capital intellectuel qui nous intéresse.

\section{B. Importance de la formation continuée}

1. Nous voudrions à présente tenter de quantifier l'effort indispensable de formation continuée du personnel chargé des responsabilités majeures que nous avons considéré ci-avant.

La question qui se pose est la suivante : pour assurer un effort de R.D. (vis-à-vis de situations courantes de production qui ont fait l'objet de nos estimations au chapitre A.) dans quelle proportion convient-il d'accroitre le capital intellectuel évoqué ci-avant?

Nous admettrons que la R.D. repose exclusivement sur les épaules des détenteurs du capital intellectuel et qu'elle consiste surtout en dépenses de personnel, ce qui est fréquemment le cas.

Nous admettrons aussi que la R.D. correspond au temps consacré en recyclage, au sens large du terme (Tout travail intellectuel orienté supplémentaire aux compétences de base).

Nous ne pouvons raisonner que sur un exemple que nous avons voulu très simple. Celui d'une firme qui s'impose un effort continu de 0,01 du chiffre d'affaire, dont les dépenses de personnel sont de $50 \%$ de ce chiffre et dont le personnel détenteur du capital intellectuel émarge financièrement pour $10 \%$ de la masse salariale totale.

Dans ces conditions, l'effort de R.D. devra être assumé par les détenteurs du capital intellectuel dans la proportion de

$$
\frac{0,01}{0,5 \times 0,1}=0,2
$$

Cet effort sera donc représenté par un surcroît de compétences intellectuelles à assurer (représenté théoriquement par le diagramme de la figure 11), vis-à-vis de celles représentées aux figures 7 et 8 . Cet effort conduit donc aux diagrammes des figures 12 et 13, (voir page suivante).

On peut en déduire les diagrammes suivants d'occupation journalière dont ressortent les heures à consacrer à la "formation" au sens large du terme (fig. 14 et 15).

Des diagrammes de principe de ce genre ont été présentés en 1985 lors de la séance organisée au F.N.R.S. sur la formation continuée. 


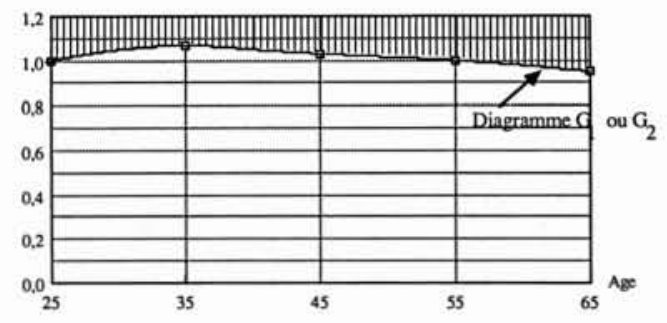

11. Surcrôt de valeur intellectuelle à assurer.

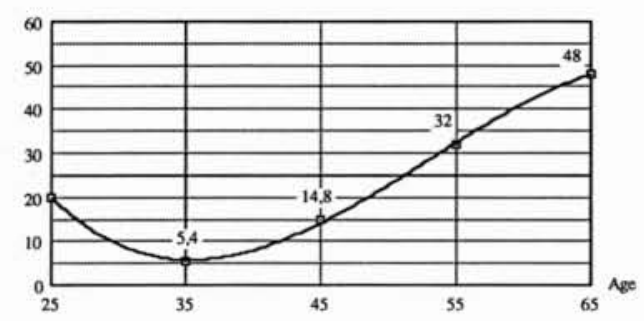

12. Surcroît de compétences intellectuelles à assurer. (Cas d'activités traditionnelles.)

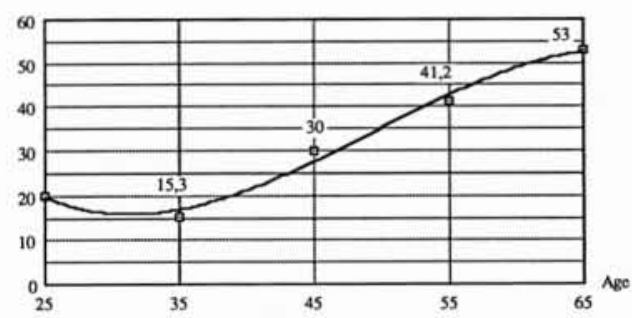

13. Surcroît de compétences intellectuelles à assurer. (Cas d'activités récentes.)

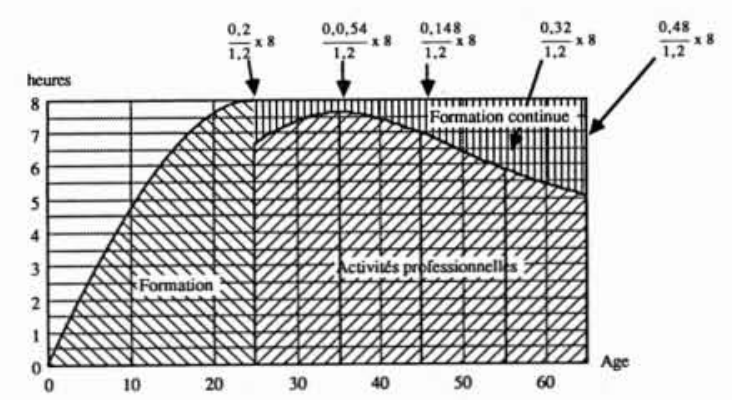

14. Estimation des nombres d'heures consacrées à la formation ( $y$ compris recyclage) dans le cas de secteurs traditionnels.

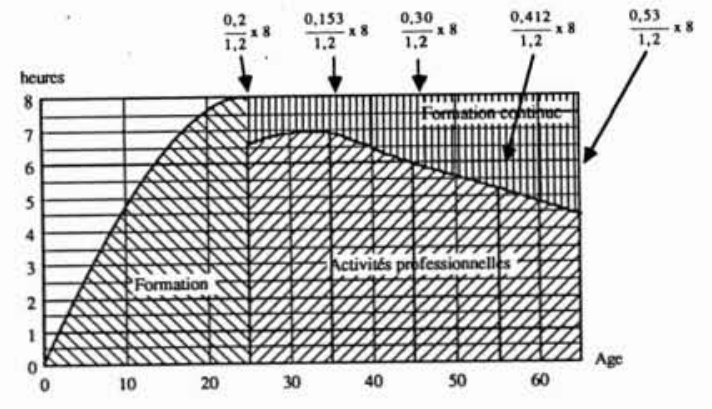

15. Estimation des nombres d'heures consacrées à la formation (y compris recyclage) dans le cas de secteurs récents.

\section{Conclusions}

Le capital immatériel des entreprises est considérable. II devrait faire l'objet dans chaque firme d'une quantification et de la prise de conscience de son importance.

L'entretien et l'amélioration de ce capital postulent des efforts importants de formation continuée.

Rappelons que les considérations chiffrées précédentes n'ont qu'une valeur illustrative sans prétention à la rigueur. Elles ne sont qu'un guide de réflexion «matématoïde » que chacun pourra poursuivre et critiquer.

\section{La formation continuée en hydraulique et les perpectives du $3^{\mathrm{e}}$ millénaire}

Après avoir montré, du moins je l'espère, l'importance de la formation continuée je voudrais exposer quelques considérations sur ce dont doit être faite la post-formation d'un ingénieur hydraulicien.

Je vous prie d'avance d'excuser le caractère personnel qu'elles peuvent avoir. Elles reposent sur l'expérience de 30 ans d'existence d'une institution liée à l'Université de Liège: (LHCN - Laboratoire d'hydraulique, d'hydraulique appliquée, de construction hydraulique et d'architecture navale).

A. D'une part, la formation continuée ayant pour but la R.D. doit conduire à l'éclosion d'idées nouvelles ou de concepts innovants, d'autre part l'hydraulique n'est que rarement une fin en soi (pièces d'eau de Versailles derrière lesquelles se cache l'extraordinaire machine de Marly due d'ailleurs au génie du liégeois R. Sualem). 
L.H.C.N. (1962)

\section{- UNIVERSITE DE LIEGE}

- Ingénieur en $2+3$ ans (Ingénieur des Constructions)

- $3^{\mathrm{e}}$ année

$100 \mathrm{~h}$

- $4^{c}$ année

$150 \mathrm{~h}+$ option hydraulique $(170 \mathrm{~h})$

- $5^{\mathrm{c}}$ année

$140 \mathrm{~h}-$ option hydraulique $(150 \mathrm{~h})$

+ T.F.E.

- Maîtrises et doctorats

2 à $3 / \mathrm{an}$

- 35 personnes dont $12 \mathrm{Ph}$. D. (8 nationalités)
- Recherches fondamentales (labo + mathématique)

- Recherches appliquées (quasi essentiellement pour étranger)

- Trois laboratoires dont un bassin de carènes de $100 \mathrm{~m}$

- Enseignement pour P.V.D. $\rightarrow$ IFIT (Voies navigables)
B. Au contraire, l'hydraulique est une connaissance de base au service de la construction hydraulique en général (barrages, écluses, machines, constructions navales, off-shore, ...) et c'est bien là que les idées nouvelles ont intérêt à se manifester.

C. Pour qu'il y ait un tissu favorable, il importe donc que les acteurs soient conscients à la fois des problèmes de l'hydraulique et des problèmes de construction :

Exemple : La Revellata

Porte marée-tempête d'Oosterweel Sines

C'est une difficulté majeure pour les hydrauliciens purs qui jouent sur des modèles et risquent de perdre le sens des réalités.

C'est une difficulté majeure pour les constructeurs purs qui sont souvent très éloignés des notions de base d'hydraulique.

Un premier défi est donc de former des constructeurs hydrauliciens ( $1^{\text {re }}$ forme de bidisciplinarité).

D. Il importe en outre que l'acteur suppute l'exacte dimensions des choses: il devra donc faire des projets de voies navigables, de lignes d'eau, de barrage, ... quelques pénible que ce soit: l'enseignement strictement limité à ce qui se dit dans les auditoires peut dans notre domaine engendrer de graves erreurs

Exemple: Similitude de Reynolds en construction navale.

E. Il est indispensable que l'enseignement soit épaulé par des démonstrations et des interventions personnelles directes dans un laboratoire de quelqu'importance où l'on vit les problèmes de tous les jours.
Le ressaut pour être compris nécessite visualisation car pour le "vulgum pecus » il est contre nature.

Nous avons reçu récemment à l'U.Lg. la visite d'ingénieurs étrangers réputés hydrauliciens qui n'avaient jamais vu une ligne d'eau, ni un déversoir de leur vie. On ne peut escompter d'eux qu'ils soient créatifs.

F. Il me paraît plus que souhaitable que l'ingénieur hydraulicien soit informé des notions relevant de la construction navale : la préfabrication, le remorquage et l'immersion sont de nos jours fréquemment pratiqués et la stabilité du flotteur doit être bien comprise.

Ainsi, il m'a souvent été dit qu'une porte busquée d'écluse ne pourrait être employée que pour des sas étroits. L'adjonction d'un caissaon sous flottaison modifie complètement cette asertion.

(seconde forme de bi-disciplinarité).

Cette seconde bi-disciplinarité s'impose évidemment si l'on veut apporter du neuf dans des domaines connexes tels celui du génie sanitaire où les connaissances en hydraulique doivent être associées de connaissances en chimie, biologie et bactériologie, ou encore celui de l'hémodynamique où de sérieuses notions de physiologie s'imposent.

G. Combien n'avons nous pas vu de thèses qui sont autant de pièces de bravoure mathématicoinformatiques où se cotoient Eléments finis et Eléments frontières. Un engouement prolongé pour ces travaux risque de stériliser les esprits : ces travaux expliquent et c'est déjà très bien mais une polarisation par trop cartésienne peut faire perdre de vue les notions fondamentales importantes.

Un problème aujourd'hui est que certains jeunes hydrauliciens portés par la vague du génie informatique 
pensent que pour faire sérieux, il faut passer plus de temps devant un ordinateur qu'au contact de la réalité des constructions.

Ils perdent ainsi petit à petit le contact intime, je dirais presque physique, qu'ils doivent avoir avec la nature, le béton et l'acier.

Ils oublient petit à petit que si l'optimisation exige un bagage scientifique solide, il reste malgré tout un art, un métier d'artisan, avec toute la dose de modestie que cela implique...

Voilà pour moi quelques idées forces ; l'hydraulique a un formidable avenir devant elle mais à condition d'être humble et d'entrer en communion de pensée avec ceux qui s'en servent.

H. Je voudrais à présent aborder un autre sujet qui me tient à cœur et pour lequel j'ai écrit pour l'association des ingénieurs sortis de l'U.Lg. un article intitulé Afrique 1992.

Je suis depuis de nombreuses années sensibilisé au problème du développement de ce continent car je ne crois pas à une coexistence pacifique entre 400 millions d'Européens repus et 400 millions d'Africains affamés.

L'Afrique est pour nous un fantastique défi à relever et tout y repose évidemment sur la source vitale qu'est l'eau : alors que certains jours le Zaïre provoque des inondations, le Mali est en état de sécheresse quasi permanente.

Bien sûr, nous aurons à écoler des ingénieurs hydrauliciens d'Afrique pendant encore de nombreuses décennies (les laboratoires d'hydraulique d'Afrique membres de l'A.I.R.H. sont au nombre de 5 dont 4 en Afrique du Sud) et nous devrons le faire dans nos institutions.

Mais l'expérience (à la fois heureuse et malheureuse) que j'ai du Zaïre m'a conforté dans l'idée qu'il faut aller dans le sens de la réalisation d'accords-cadres de région à région (d'Afrique et d'Europe) organisant une convergence des intérêts et favorisant une authentique coopération (au sens premier du terme et non au sens fallacieux qu'on lui donne de nos jours).

L'objectif est que des firmes partenaires des deux régions s'associent pour concrétiser des projets ensembles, en définir les modalités, en partager les risques et bien sûr les bénéfices.

L'Europe des Régions doit ainsi s'associer à l'Afrique des Régions. Dans cette perspective, les bassins hydrographiques sont les bases les plus logiques d'implantation parce que liées à l'économie et la culture.

Ce n'est pas du néocolonialisme c'est du bon sens de partenariat.

En 1885, l'Acte de Berlin était allé dans cette voie en s'intéressant aux bassins du Niger et du Congo. Il conviendrait d'en relire les propositions pour préparer une convention de Lomé, cinquième du nom.

I. Enfin, à mon avis, la meilleure façon de préparer notre avenir et celui de nos amis africains sera dans notre collaboration au niveau européen.

A ce sujet, il m'est agréable, à peu de distance de la Sorbonne de signaler une initiative, à laquelle j'ai participé dès 1985 et qui vise à créer ce que Mme $\mathrm{H}$. Ahrweiler actuellement présidente du centre Pompidou et à l'époque, recteur-chancelier des Universités de Paris, a appelé l'Université de l'Europe, université sans mur, regroupant les institutions européennes (universitaires ou non) capables d'apporter leur spécificité en vue d'une formation de très haute qualité.

Nous aurions, je le crois très sincèrement, un grand intérêt à y associer l'hydraulique au sens large du terme. 